\title{
Escritura e Imagen
}

ISSN: $1885-5687$

http://dx.doi.org/10.5209/ESIM.62760

\section{El pathos en el Estudio Patético de Aleksandr Scriabin}

\author{
Dario D. Aguillón Gutiérrez ${ }^{1}$
}

Recibido: 8 de julio de 2017 / Aceptado: 8 de mayo de 2018

Resumen. La música como expresión artística esconde significados que trascienden a la estética, que pueden analizarse desde otras disciplinas y campos de estudio. La retórica como arte del discurso y herramienta del lenguaje, puede encontrarse en la composición musical como elemento persuasivo, que utiliza el pathos como medio para producir emociones en el oyente. El presente texto analiza el Estudio Patético Op. 8 No. 12 de Aleksandr Scriabin desde la perspectiva del discurso, a fin de encontrar las figuras retóricas asociadas al concepto del pathos griego, utilizando como referencias análisis previos a obras musicales con el adjetivo patético. El texto también busca aportar claridad respecto al concepto de lo patético, cuyo significado ha sufrido transformaciones a lo largo de los siglos, alterando su concepción griega original. El presente análisis propone abordar las obras musicales desde la perspectiva de la retórica, a fin de lograr una mayor comprensión de la composición y una mejor interpretación por parte del ejecutante.

Palabras clave: Música; estética; discurso; retórica; pathos.

\section{[en] Pathos in Aleksandr Scriabin's Étude Patetico}

\begin{abstract}
Music as an artistic expression hides meanings that transcend aesthetics, which can be analyzed from other disciplines and fields of study. Rhetoric as the art of discourse and as a tool of language can be found in musical composition as a persuasive element, which uses pathos to produce emotions in the listener. The present paper analyzes the Étude Patetico Op. 8 No. 12 of Aleksandr Scriabin from the perspective of discourse, in order to find the rhetorical figures associated with the greek concept of pathos, using as references previous analysis to musical works with the pathetic adjective. The text also looks to provide clarity around the concept of the pathetic, whose meaning has undergone transformations over the centuries, altering its original greek conception. The present analysis proposes to approach musical works from the perspective of rhetoric, in order to achieve a greater understanding of the composition and a better interpretation by the performer.
\end{abstract}

Keywords: Music; aesthetics; discourse; rethoric; pathos.

Sumario: 1. La retórica aristotélica; 2. Del pathos a lo patético; 3. Pathos y arte; 4. La música patética; 5. El Estudio Patético; 6. Conclusión; 7. Referencias bibliográficas.

Cómo citar: Aguillón Gutiérrez, D.D. (2018) "El pathos en el Estudio Patético de Aleksandr Scriabin", en Escritura e Imagen 14, 45-63.

1 Universidad Autónoma de Coahuila.
dario.aguillon@gmail.com 


\section{La retórica aristotélica}

Es un hecho innegable que la cultura griega es la base y cimiento de toda la cultura occidental vigente hasta nuestros días. Todo el saber humano fue sintetizado por los griegos de tal manera que fue posible categorizar el conocimiento, además de empezar una larga historia que después permitiría no sólo incrementar ese conocimiento, sino comprender como el ser humano tenía la posibilidad de adquirirlo, es decir, de conocer. Esa larga historia y ese antiquísimo esfuerzo de categorizar el conocimiento fue llamado filosofía, y constituyó el inicio del entendimiento de todo cuanto hay en la vida del hombre, incluyendo su entorno, los objetos con los que interactúa, sus acciones y su pensamiento.

Si bien Sócrates desarrolló la mayéutica y Platón la dialéctica, Aristóteles estructura y forma un nuevo método filosófico al que llama "lógica", que consiste en la aplicación de las leyes del pensamiento racional que nos permite encontrar relaciones entre conceptos generales para llegar después a conceptos particulares. ${ }^{2}$ La lógica como disciplina que estructura el pensamiento se desarrolla en el contexto social de la antigua Grecia, y pronto requiere nuevas disciplinas o ramas que faciliten la transmisión o expresión de ese pensamiento razonado. La necesidad de argumentación en base al contenido, a las pruebas y evidencias con fines de persuasión, permite el nacimiento de la retórica como rama de la lógica que organiza y sistematiza el discurso.

En su Retórica, Aristóteles describe con profundidad el método de persuasión como herramienta de la dialéctica, y describe a la retórica como "la facultad de teorizar lo que es adecuado en cada caso para convencer". ${ }^{3}$ Si bien puede ser considerada un arte, una técnica o incluso una ciencia, su función en el discurso presenta una marcada diferencia con la dialéctica: mientras que la dialéctica busca conclusiones racionales, la retórica busca además una empatía emocional con la audiencia. ${ }^{4}$

Aristóteles define que el objetivo esencial de la retórica es "convencer, persuadir" y para él, son tres las formas en las que el orador puede lograr dicho objetivo. La primera de estas formas, el ethos, consiste en la confianza que el orador inspira. Aristóteles dice que "se persuade por el talante, cuando el discurso es dicho de tal forma que el orador es digno de crédito, porque a las personas honradas les creemos más y con mayor rapidez". ${ }^{5}$ La segunda de ellas, el pathos, consiste en las pasiones y emociones generadas en el oyente, ya que "se persuade por la disposición de los oyentes, cuando éstos son movidos a una pasión por medio del discurso. Pues no hacemos los mismos juicios estando tristes que estando alegres, o bien cuando amamos que cuando odiamos". ${ }^{6}$ Finalmente, el $\log o s$, la tercera forma de persuadir, consiste en los argumentos, el razonamiento y el mensaje, a fin de persuadir por medio de la razón. Como lo expresa Aristóteles, "se persuade por el discurso, cuando les mostramos la verdad, o lo que parece serlo, a partir de lo que es convincente

\footnotetext{
García Morente, M., Lecciones preliminares de filosofía, México, Porrúa, 1992, pp. 26-30.

Aristóteles, Retórica. I, 1355b, Madrid, Gredos, 1990, p. 173.

4 Emanuel, B., Rodrigues, C. y Martins, M. «Rhetoric of Interaction: Analysis of Pathos», en Design, User Experience, and Usability, 1 (2015), pp. 418.

Aristóteles, Retórica. op.cit., I, 1356a, p. 176.

Ibidem, p. 177.
} 
en cada caso". ${ }^{7}$ De acuerdo entonces a la retórica clásica, el ethos se enfoca en los atributos del orador, el pathos en las emociones de la audiencia y el logos en el mensaje y el razonamiento para construir argumentos. ${ }^{8}$

\section{Del pathos a lo patético}

En el Diccionario de Retórica y Poética se define al pathos como:

Un estado afectivo más intenso. Es la conmoción que sacude al espectador de la tragedia, al lector de la epopeya, o al público que escucha la peroración -la dispositio que ordena la inventio-, del orador; la conmoción que hace llorar u horrorizarse, que obliga al juez a emitir un fallo favorable. ${ }^{9}$

Las pasiones a las que hace referencia Aristóteles cuando define el pathos como forma de persuasión, son analizadas en el Libro II de su Retórica, y las describe como "las causantes de que los hombres se hagan volubles y cambien en lo relativo a sus juicios, en cuanto que de ellas se siguen pesar y placer". ${ }^{10}$ La Retórica presenta un estudio sobre la ira, la calma, el amor, el odio, el temor, la confianza, la vergüenza, la compasión y la envidia, entre otras pasiones, a fin de demostrar la importancia de la actitud del público oyente del discurso. Aquí Aristóteles presenta una diferencia entre el ethos, la actitud del orador, la confianza y benevolencia que inspira, y que resulta más útil en las deliberaciones, y el pathos, las emociones y actitudes de los oyentes, más útiles en los procesos judiciales. ${ }^{11}$

El concepto de pathos como elemento del discurso ha sido estudiado por diversos autores, como la investigadora Luisa Puig, quien en su artículo «Del pathos clásico al efecto patémico en el análisis del discurso» ${ }^{12}$ hace un análisis del pathos desde un enfoque puramente lingüístico y su papel en la interacción comunicativa y concluye que las emociones son parte fundamental de la racionalidad y de la interacción entre los seres humanos. Derivado de esto, introduce la noción de efecto patémico, que consiste en el desencadenamiento de las emociones a través del uso de palabras específicas-, y abre un abanico de posibilidades para aplicar un análisis sobre distintos lenguajes o formas de expresión.

De acuerdo con el Compendio de Etimologías Grecolatinas del Español el vocablo griego pathos significa sentimiento, pasión, enfermedad. Describe brevemente la evolución semántica del término, que "pasó luego a significar una cierta clase de sentimiento, la pasión. Finalmente, cuando se estimó que la pasión viene a ser una enfermedad del alma, que nubla la razón, dicho vocablo acabó significando enfermedad". ${ }^{13}$ De aquí parten los términos patología, patógeno, antipatía, psicopatía, y patético. Este último término, sobre los demás, es el que podemos

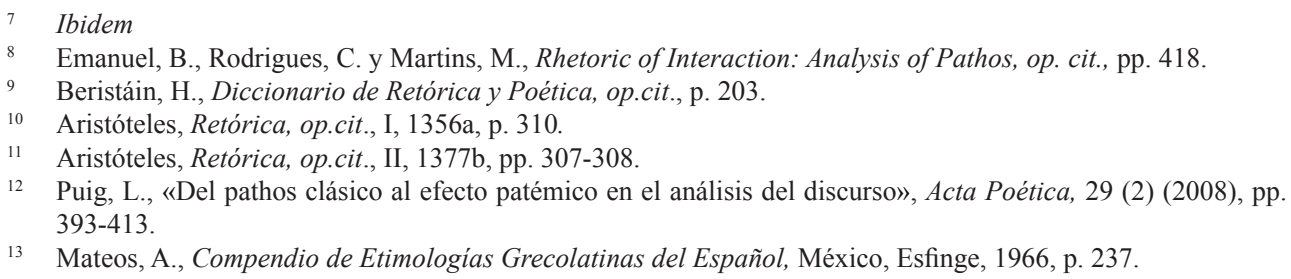


encontrar como adjetivo añadido a obras musicales. El mismo Compendio define lo patético como algo "relativo a la sensación vehemente; sobre todo de tristeza, de dolor o de melancolía". ${ }^{14}$

A pesar del claro origen del término, actualmente existe un uso mucho más habitual del término patético como un adjetivo negativo, una descalificación asociada a lo ridículo, que otorga al adjetivo un carácter peyorativo contrapuesto a su sentido original. Sorprendentemente, el origen de esta acepción negativa se encuentra en 1672, en la obra Les Femmes Savantes (Las Mujeres Sabias), del célebre dramaturgo francés Jean-Baptiste Poquelin "Molière". A partir de un diálogo en la tercera escena del tercer acto de la mencionada obra, en la que a manera de insulto y por su similitud fonética se utiliza el término pathos en sustitución del término pataud, cuya traducción al español es el adjetivo torpe, se inicia una historia del término pathos, y por consiguiente de lo patético, como una asociación a lo cómico, lo ridículo y lo banal.

El análisis de esta transformación semántica lo hace a profundidad la investigadora de la Universidad de Poitiers, Anne Coudreuse, en su artículo Pathos et pathétique au XVIII siècle: étude lexicale et statistique, ${ }^{15}$ que además presenta evidencias al analizar ediciones de 1690 a 1702 del Dictionnaire universel de Antoine Furetière y del Dictionnaire de l'Academie, que presentan por vez primera los términos pathos $\mathrm{y}$ pathétique, y asocian este último término a lo cómico y a la bufonería a partir del ejemplo de la obra de Molière. A partir de su análisis, Coudreuse afirma después que "lo patético es un término de crítica y de estética, que se convertirá en el curso de su evolución en sinónimo de "dramático", que en el uso contemporáneo no hace ya referencia al teatro, pero significa "triste, irrisorio, lamentable"". ${ }^{16}$ El estudio lexicológico que hace la autora concluye con la siguiente hipótesis:

Lo patético es quizá lo que sigue siendo legible y aceptable del pathos. Lo patético sería entonces una forma leve de pathos, que ha perdido sus excesos característicos para centrar su efecto emocional. ${ }^{17}$

Es a partir de esta acepción del término patético, más cercana a su derivación del pathos griego y a su significado semántico emocional, que se pretende encontrar su relación con las obras musicales que han recibido - por decisión o no del compositoreste adjetivo o subtítulo.

\section{Pathos y arte}

Con el desarrollo de las artes, el concepto de pathos ha sido utilizado no solo en el lenguaje escrito, sino también en el visual y musical. Es posible encontrar el pathos en una obra pictórica o en una obra musical, sin embargo, es preciso determinar a través del análisis de la obra si el concepto se utiliza en su acepción original o solo se añade el adjetivo patético sin mayor profundidad filosófica. Para determinar lo

\footnotetext{
Ibidem

Coudreuse, A., «Pathos et pathétique au XVIII ${ }^{\mathrm{e}}$ siècle: étude lexicale et statistique», Studi francesi, 126 (1998).

Ibidem, p. 13.

Ibidem, p. 15.
} 
anterior, es necesario partir de la premisa de que el lenguaje del arte se rige bajo los principios de la estética. En la teoría general del arte desarrollada por los griegos, se establece el concepto de la imitación o mimesis como medio para llegar al ideal estético en una obra y así llevar al placer a quien la admira. La mimesis como imitación de la realidad de la vida puede realizarse con fines científicos o artísticos, a los cuales corresponde el discurso literario y la poesía. Helena Beristáin explica el término en su Diccionario de Retórica y Poética:

Por medio de la mimesis, según Aristóteles, el poeta copia la realidad del mundo que lo rodea; en su obra transfunde esa realidad, por lo que su obra constituye un mimema. [...] Pero no solo existen las artes poético-miméticas que se expresan a través de la lengua, sino también otras artes miméticas que se manifiestan a través de otros sistemas de signos perceptibles por diversos sentidos, como la pintura, la música, la escultura o la danza. De esta última opina Aristóteles que "imita, con el movimiento rítmico del cuerpo, el mundo humano en general. ${ }^{18}$

Lewis Rowell reflexiona sobre la mimesis en la música en su Introducción a la filosofía de la música haciendo énfasis en la notable omisión que Aristóteles hace de la música en la lista de las artes miméticas, y se pregunta si realmente la música consiste en una imitación, que necesariamente requiere de una semejanza entre el modelo y la representación. Más allá de la mimesis como método del artista, Rowell describe el carácter de la música y lo asocia con los efectos que produce cuando el oyente la percibe. El autor asocia este concepto de carácter con el ethos, al otorgarle principalmente un sentido de carácter moral y bajo la premisa de que a la música se le asignó un papel central en la formación del carácter, siendo el moral prioritario en la educación griega. ${ }^{19}$

Es a partir de esta conexión entre la música y la formación del carácter cuando surgen las primeras premisas respecto a las emociones que se producen en el oyente. Damón de Atenas dice que "el canto y la danza surgen necesariamente cuando se conmueve de alguna forma al espíritu", mientras que Aristóteles dice que "algunas (escalas musicales) entristecen y afiebran a los hombres y los hacen sentirse graves, como las llamadas mixolidias; otras afiebran la mente...Los mismos principios aplican a los ritmos". ${ }^{20}$ De acuerdo con estas ideas podemos destacar el poder de la música para la formación del carácter de los ciudadanos griegos, a quienes, dependiendo de su estado de ánimo o salud mental, se les sometía a una especie de terapia musical que les equilibrara. El manejo de las pasiones a través de la música permite el surgimiento de otros conceptos, de entre los cuales figura la katharsis, que Aristóteles introdujo en su Poética y denomina como "el efecto purificador de las pasiones (temor, odio, compasión) que producen en el receptor la poesía y el drama, especialmente la tragedia". ${ }^{21}$ En general el término se utilizó para referirse a esa purificación, esa liberación del exceso de emociones de quien ha vivido la experiencia del drama trágico, o la experiencia de la música.

\footnotetext{
Beristáin, H., Diccionario de Retórica y Poética, op.cit., p. 336.

Rowell, L., Introducción a la filosofia de la música, Buenos Aires, Gedisa, 1985, p. 58.

Ibidem.

Beristáin, H., Diccionario de Retórica y Poética, op.cit., p. 91.
} 
Sin duda el arte ha cumplido con esta función catártica a lo largo de toda su historia, ya sea a través de la contemplación de una obra pictórica con técnica impecable, una escultura religiosa en un templo sagrado, o una pieza musical de belleza indescriptible. Es innegable la capacidad de la música de producir emociones y de mover el espíritu de quien la escucha. El ritmo, el tempo, la melodía y la armonía como elementos de la mimesis del artista son capaces de producir reacciones en el cerebro del oyente que le llevan a experimentar toda una gama de sensaciones y sentimientos. Existen diversos estudios sobre los efectos de la música en el cerebro, y como a través de ella es posible sanar y equilibrar no sólo la mente sino también el espíritu.

Si bien es evidente la relación entre el pathos discursivo y la creación musical, es necesario preguntarse cuál es la forma y herramientas que utilizan los compositores para la construcción de su discurso. En la historia de la música, es inmensa la cantidad de adjetivos o subtítulos añadidos a las obras, que buscan otorgarle un carácter específico y así contextualizar al intérprete para que transmita el mensaje de la forma deseada, y al oyente para aportar a la comprensión de lo que escucha. Podemos encontrar innumerables ejemplos de obras fantásticas, melancólicas o tristes, alegres, apasionadas, trágicas, heroicas, y por supuesto, patéticas, que, sin ser necesariamente de carácter descriptivo o programático, añaden una personalidad propia a la obra, que resulta enriquecida y no limitada a la teoría y la técnica compositiva.

La idea de asociar el carácter de la música a un sentimiento específico se desarrolla y se hace presente en épocas como el renacimiento y el barroco. En esta última, la asociación entre música y sentimientos queda plasmada en la Teoría de los Afectos, desarrollada a partir de las ideas filosóficas de Descartes y estudios de compositores que buscaban ampliar el lenguaje de la música. Uno de ellos, Johann Mattheson (1681-1764), aportó a ello a través de su entendimiento de los intervalos y sus propiedades afectivas, y sus estudios, tratados y composiciones influyeron notablemente en los músicos de su generación. ${ }^{22}$ Además del estudio de intervalos, el estudio de las tonalidades también contribuyó a la Teoría de los Afectos por medio de las obras de compositores como Jean Philippe Rameau, que encontraron propósitos expresivos en las tonalidades y sus distintos modos. Por ejemplo, para Rameau la tonalidad de Mi mayor representa "grandeza y magnificencia", mientras que Fa menor es la tonalidad asociada al invierno. ${ }^{23}$

Ya en el siglo XX, la relación música-emociones tuvo cabida en el expresionismo musical de la Segunda Escuela Vienesa, sin embargo, con estrecha relación con el intelectualismo de las vanguardias. Como lo expresa Arnold Schoenberg, pionero de la composición atonal y creador del dodecafonismo, citado por James K. Wright: "El mundo de los sentimientos es inseparable del mundo del intelecto; los dos se sienten siempre como uno y el mismo." ${ }^{24}$ Schoenberg complementa la idea partiendo de lo dicho por Balzac en su obra Seraphita - "El corazón debe estar bajo el dominio del cerebro"-:

\footnotetext{
22 Dissmore, J., « Baroque Music and the Doctrine of Affections:Putting the Affections into Effect», The Research and Scholarship Symposium, 18 (2017), p. 3.

23 Ibidem, p. 9.

24 Wright, J., Schoenberg, Wittgenstein and the Vienna Circle, Berna, Peter Lang, 2007, p. 99.
} 
No es el corazón solo el que crea todo lo que es bello, emocional, patético, afectivo y encantador; tampoco es el cerebro solo el que produce lo bien construido, lo sonoramente organizado, lo lógico y lo complicado. Todo el valor supremo del arte debe mostrar tanto corazón como cerebro. ${ }^{25}$

Este dualismo en la actividad creativa equilibra el sentimiento y la emoción con el conocimiento y la técnica, ambos de gran profundidad y complejidad en la composición expresionista.

Sin embargo, es pertinente decir que la gran mayoría de melómanos no conocen los recursos técnicos utilizados por los compositores. No comprenden de movimientos armónicos, de inflexiones tonales ni de cambios rítmicos. Pero si son capaces de apreciar el valor estético de lo que escuchan, y sobre todo son capaces de sentir emoción, aceptando el discurso y la persuasión a través del pathos. De entre todas las obras con un carácter propio, son las obras que han recibido el adjetivo patético las que merecen especial atención en el contexto de la presente investigación.

\section{La música patética}

Como hemos señalado anteriormente, el adjetivo patético es tratado en el presente texto desde su acepción original derivada del pathos aristotélico, eliminando todo rastro del término cómico utilizado desde el siglo XVII y que todavía hoy tiene una connotación negativa y peyorativa. Es entonces conveniente analizar por qué ciertas obras reciben dicho adjetivo, desde su contexto histórico y desde su forma y contenido musical.

No es casualidad que las obras que aquí serán analizadas estén inscritas en el período romántico de la música, caracterizado por la exaltación de las pasiones y los sentimientos, y plagado de una libertad creadora que permitió a los compositores volcar su espíritu en su discurso musical. Sin embargo, como lo expresa Schiller en su ensayo Sobre lo patético (incluido en sus Escritos sobre estética), no debe confundirse lo patético con algo meramente sensible. ${ }^{26}$ Para él lo patético solo es estético en la medida en que es sublime, insistiendo en que lo sublime procede de la razón y sólo de ella. De esta manera Schiller añade el factor de la razón como fuerza suprasensible que permite la resistencia al padecimiento, es decir, que sólo el razonamiento como acto noble -término que el filósofo contrapone a lo vulgar-, es el que permite al hombre que su poder moral se imponga a su instinto, a su animalidad, y de esta manera puede defenderse del objeto que le hace padecer. Para Schiller lo patético no es el sufrimiento por sí mismo, sino la resistencia a él apoyada en la razón. Podemos observar como el pensamiento filosófico y estético del romanticismo recuperan y adaptan el concepto griego del pathos a su entorno, facilitando el análisis y comprensión del término patético aplicado a una obra de arte.

Dentro de las obras musicales más célebres que reciben el adjetivo patético se encuentra la Sonata para piano Op. 13 en Do menor de Ludwig van Beethoven, mejor conocida como la "Sonata Patética". Al escuchar detenidamente la obra es tan fácil identificar sus pasajes apasionados y emotivos -el Grave introductorio

Ibidem.

26 Schiller, F., Escritos sobre estética, Madrid, Tecnos, 1991, pp. 65-96. 
del primer movimiento o el Adagio cantabile del segundo-, que si hiciéramos una aproximación superficial al concepto del pathos podríamos encontrar similitudes y relación lógica entre lo que se escucha y lo que esto provoca en el ánimo del oyente. Incluso la mayoría de los biógrafos de Beethoven no busca un significado más profundo del título de la obra, y sólo la mencionan como una sonata de importancia que representa una transición entre las obras de juventud y el período heroico de la música beethoveniana. Uno de los biógrafos del compositor alemán de mayor prestigio, Maynard Solomon, sólo refiere que la Sonata Patética "ha gozado de gran popularidad en parte a causa de su título romántico, pero ello no disminuye su verdadera importancia. Hasta aquí es de todas las sonatas para piano de Beethoven la que exhibe energía más dinámica, la primera sonata que utiliza una introducción lenta y dramática, y la primera cuyos movimientos aparecen clara e inequívocamente vinculados mediante el empleo del material temático afín y las reminiscencias conscientes" ${ }^{\prime 27}$. Este fragmento constituye una opinión superficial respecto al título de la obra, que, como ya se ha dicho, no busca un significado profundo ni mucho menos una relación con el concepto griego del pathos. Esto es comprensible ya que el objetivo del texto de Solomon es presentar una biografía completa de la vida de Beethoven, y no un análisis de sus obras y su significado.

Afortunadamente la obra del genio de Bonn ha sido sujeta a investigaciones tan específicas que no se limitan a su vida o al análisis musical desde un enfoque teóricotécnico, sino que buscan estos significados y relaciones con el pensamiento filosófico y el contexto histórico en que surgen sus obras. Una de estas investigaciones es la realizada por Elaine R. Sisman, en su artículo titulado Pathos and the Pathétique: rethorical stance in Beethoven's C-minor sonata, Op. 13, que aborda precisamente la relación entre el título de la sonata y el concepto griego del pathos. Sisman introduce al tema contextualizando respecto a la importancia de la retórica en el siglo XVIII, y como se manifiesta en distintas artes, específicamente en el arte de escribir cartas o ars dictaminis, que cultivaron compositores como Haydn, Mozart y el propio Beethoven. De este último, queda como evidencia su Testamento de Heiligenstadt, que constituye una obra literaria de un profundo tono emocional y llena de expresiones apasionadas que nos permiten encontrar un verdadero pathos del discurso.

Es importante recordar que el conocimiento que tenemos sobre la trágica vida de Beethoven lo debemos a su correspondencia, en la que el compositor acostumbraba plasmar sus sentimientos y pasiones y que afortunadamente fueron conservadas por sus destinatarios. Para encontrar la relación de la Sonata Patética con el pathos, Sisman hace un breve análisis de la utilización del concepto de pathos en el siglo XVIII, y encuentra que la tendencia entre los escritores europeos es utilizar el pathos con una acepción alterada respecto a su significado original. Para ellos, el concepto de ethos representa una calma de fondo, continuidad y estabilidad, mientras que pathos representa a las emociones fuertes que mueven las pasiones de quienes escuchan el discurso. ${ }^{28}$

Pathos entonces se asocia comúnmente a las emociones fuertes, elevadas, cercanas a lo sublime. Incluso en el Musikalisches Lexikon (Diccionario musical) de

\footnotetext{
Solomon, M., Beethoven, Buenos Aires, Javier Vergara, 1983, p. 138.

28 Sisman, E., «Pathos and the Pathétique: retorical stance in Beethoven's C minor sonata Op. 13», Beethoven Forum, vol. 3 (1994), pp. 88.
} 
Heinrich Christoph Koch, ${ }^{29}$ publicado en 1802, ambos términos, patético y sublime, aparecen como sinónimos, mientras que Johann Christoph Adelung, ${ }^{30}$ en su Über den Deutschen Styl (Sobre el estilo alemán), describe cuatro estilos que se diferencian de acuerdo con su intensidad y elevación: el estilo familiar, el estilo que conmueve, el estilo patético y el estilo sublime. Adelung refiere que lo patético son las grandes emociones y pasiones, pero que cuando su fuerza y tamaño es inusual y maravillan y sorprenden a la audiencia, entonces se transforma en lo sublime.

Esta idea se torna interesante cuando salen a la luz las figuras retóricas que en la época eran asociadas al pathos, y sobre todo porque estas figuras -la antitesis, la exclamación, la corrección, la hipérbole y la prosopopeya, entre otras utilizadas principalmente en la literatura- encontraron sus equivalentes en el lenguaje musical. Sisman refiere que teóricos de la música de los siglos XVII y XVIII, como Burmeister, Bernhard y Walther, encontraron estas equivalencias relacionadas al estilo patético en figuras musicales como la pathopoeia-semitonos cromáticos-, el passus duriusculus -movimiento descendente de semitonos-, y el saltus duriusculus -saltos disonantes de al menos una sexta, o intervalos aumentados o disminuidos-. Esta última figura retórica musical tiene relación con el estilo patético de la armonía descrito por Jean Jacques Rousseau, que consistía en una mezcla de acordes aumentados y disminuidos, en tonalidades con poco en común para que se produzca la disonancia y perturben el oído. Incluso la tonalidad de Do menor, junto con Sol menor y Fa menor, era considerada una tonalidad patética.

Probablemente no sea una casualidad que la Sonata Patética de Beethoven esté compuesta precisamente en la tonalidad de Do menor. Si bien es más fácil relacionar las tonalidades menores con sentimientos como la melancolía, la ira o la desesperanza, asociados comúnmente al pathos, no necesariamente todas las obras compuestas en este modo poseen el carácter patético. Sería superficial y aventurado afirmarlo así. Para adquirir este carácter, es necesario utilizar las figuras retóricas correspondientes asociadas al pathos, que de acuerdo al análisis de Sisman, se encuentran en la Sonata Patética: $:{ }^{31}$ el acento patético, que consiste en un acorde de séptima disminuida, cierra la primera frase del Grave del primer movimiento; la siguiente frase se presenta a manera de interrogación (realización de una pregunta retórica); posteriormente una corrección (cambio de una palabra por otra de mayor impacto, para lograr la atención) que nos conduce a un cambio de tonalidad, en este caso a la relativa mayor (mi bemol mayor), que cambia el color y la textura de la pieza y la dirige a una zona de contrastes dinámicos y modales; en esta nueva tonalidad, se sucede una gradación (crecimiento e intensificación hacia un clímax) a manera de progresión dirigida por octavas en la melodía, y apóstrofes que conducen a una hipérbole, es decir, una exageración, una amplificación, no sólo en el aspecto dinámico sino también en la posición geográfica de las octavas; el crescendo conduce a un clímax que es sucedido por prosopopeyas (esta figura tiene un doble significado, y aunque Sisman la utiliza en el sentido de la personificación, también puede analizarse desde el de la gravedad y la solemnidad) para finalmente cerrar el Grave con una exclamación (figura que puede representar admiración o dolor), es decir, un salto de séptima disminuida descendente (figura 1). ${ }^{32}$

29 Ibidem, p. 93.

30 Ibidem, pp. 94-95.

31 Sisman, E., Pathos and the Pathétique: retorical stance in Beethoven's C minor sonata Op. 13, op. cit., pp. 99 105 .

32 Ibidem, p. 100. 

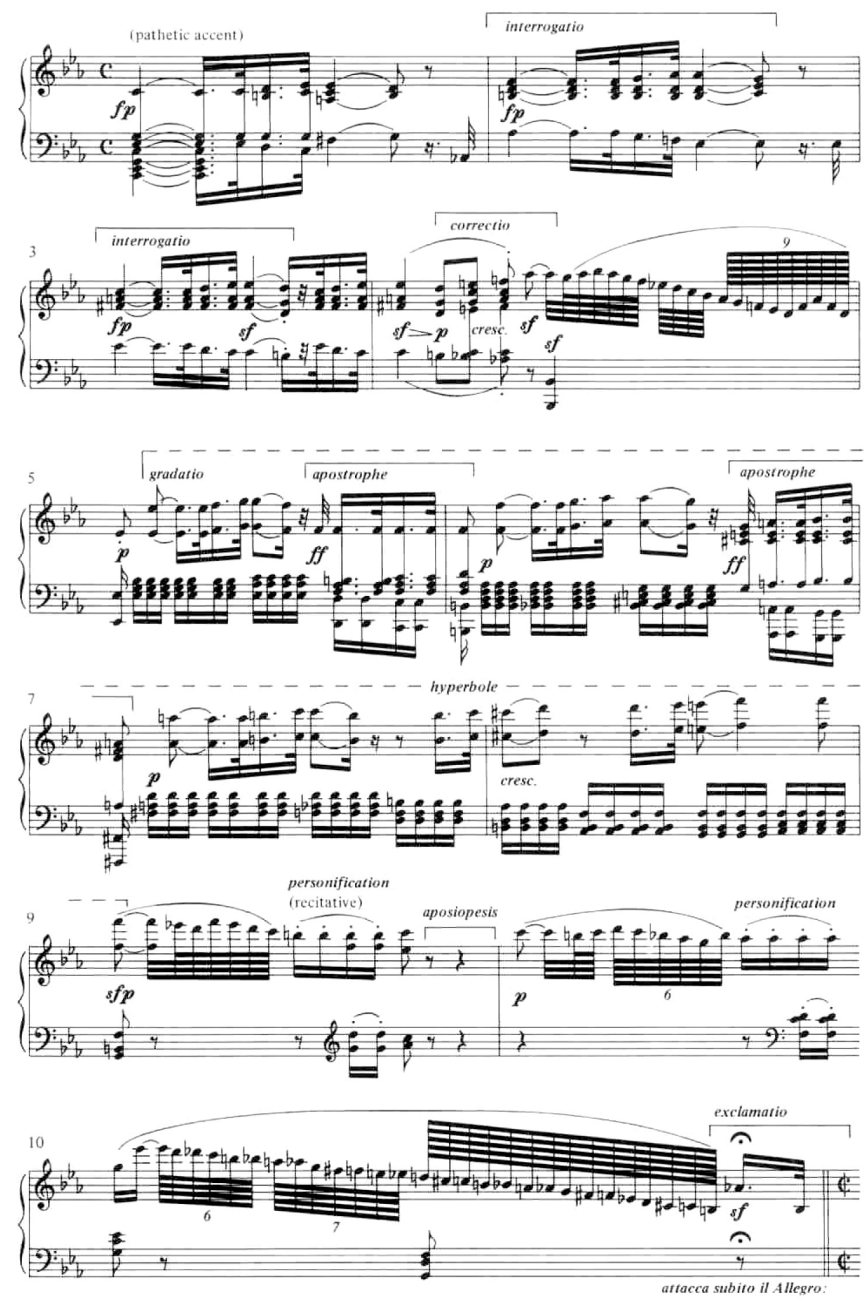

Figura 1. Análisis del Grave de la Sonata Patética de Beethoven, realizado por Elaine R. Sisman.

A lo largo de toda la sonata, según el análisis de Sisman, se desarrolla una lucha, una resistencia al sufrimiento representada en el Allegro del primer movimiento, con retornos de las ideas musicales del Grave, que se alternan a manera de diálogo y que rompen la continuidad del Allegro (recordemos que en la época el pathos representaba lo breve, mientras que el ethos representaba lo continuo y estable). El resto de la obra contiene visiones de lo expuesto en el primer movimiento: mientras que el Adagio cantabile es una pieza de gran emoción, el Rondó recupera uno de los temas del primer movimiento y finaliza con una visión (o fantasía, la vívida imagen de una escena ausente) $)^{33}$ de las escalas descendentes del Grave.

33 Sisman, E., Pathos and the Pathétique: retorical stance in Beethoven's C minor sonata Op. 13, op. cit., pp. 96- 
Sin duda la investigación de Sisman es de especial relevancia al abordar el concepto de lo patético no sólo desde un enfoque filosófico e histórico referencial, sino también desde la teoría y el análisis puramente musical, lo que enriquece y aporta al entendimiento de la obra. Aunque no hay certeza de quien asignó el adjetivo "patética" a la sonata en Do menor -algunos historiadores afirman que fue una acertada decisión del editor, con la aprobación de Beethoven-, el análisis al que ha sido sometida nos permite deducir que probablemente ningún otro adjetivo hubiese sido más apropiado.

Si bien se pueden formular hipótesis respecto a la composición consciente de la Sonata Patética, en el sentido de que Beethoven era un asiduo lector de la filosofía griega, así como de Schiller y otros filósofos alemanes, y por consiguiente se puede considerar posible que haya tenido en cuenta el concepto del pathos y lo patético a la hora de componer la sonata en Do menor, no ocurre lo mismo con la Sinfonía Patética de Piotr Ilyich Tchaikovsky.

Obra fundamental del repertorio sinfónico del romanticismo tardío, la Patética fue compuesta en 1893 y estrenada el 16 de octubre del mismo año, nueve días antes de la muerte del compositor. Gracias a la correspondencia que aún se conserva, - y que fue recopilada por Alexandra Orlova en el volumen Chaikovsky: un autorretrato-, es posible saber que Tchaikovsky no asignó el adjetivo "patética" a su sexta sinfonía, y que no fue compuesta con la intención de relacionarla con el concepto filosófico del pathos. La idea original que el compositor tenía para su última sinfonía quedó plasmada en una carta enviada por él a su sobrino y amigo Vladimir Lvovich Davidov:

Mientras estaba de viaje, tuve una idea para otra sinfonía, esta vez una sinfonía con programa; pero ese programa será un enigma - que lo adivine la gente-, y la obra se llamará realmente Sinfonía con Programa (número 6). Este programa es tan intensamente personal que mientras lo componía mentalmente en mis viajes lloraba copiosamente. ${ }^{34}$

El carácter personal de la obra al que se refiere es el que probablemente motivó a su hermano Modest a añadirle el título Patética un día después de su estreno, al encontrar una relación entre el concepto de lo patético como sinónimo de lo emotivo y apasionado, y la vida del compositor. Incluso la sinfonía llegó a ser conocida como "la sinfonía del suicidio", por la cercanía entre las fechas de su estreno y la muerte de su autor. ${ }^{35}$ La vida apasionada y trágica del artista encuentra eco en su obra, que además es de una calidad musical e innovación formal que le valió un especial aprecio del compositor, que la consideraba su mejor obra.

Los ejemplos anteriormente expuestos presentan diferencias respecto a su relación con el concepto del pathos griego. El primero de ellos se relaciona más íntimamente al considerar en su composición elementos formales asociados a lo patético, como la tonalidad, las figuras retóricas y el carácter de lucha y resistencia al sufrimiento descrito por Schiller. El segundo ejemplo, si bien no tiene una relación más allá del nombre, también es una obra de carácter apasionado, aunque será más probable encontrar relación con la vida del compositor que con el concepto del pathos aquí expuesto.

34 Orlova, A., Chaikovsky: un autorretrato, Madrid, Alianza, 1994, p. 417.

35 Gutiérrez Heras, J., Notas sobre notas, México, CONACULTA, 1998, pp. 140-141. 
La tercera obra por analizar comparte nombre y carácter con los ejemplos anteriormente analizados: se trata del Estudio Op. 8 No. 12 "Patético" en re sostenido menor del compositor ruso Aleksandr Nikoláyevich Scriabin, obra fundamental del repertorio pianístico posromántico. Sin embargo, es preciso determinar qué tan profunda es la relación con el pathos y si el adjetivo "patético" le fue añadido por razones filosóficas y formales o sólo por una asociación superficial a la emoción y las pasiones.

\section{El Estudio Patético}

La obra de Scriabin puede dividirse en dos épocas, de acuerdo con las influencias recibidas no sólo en lo musical sino también en lo filosófico. La primera época, a la que corresponden las obras compuestas entre 1886 - año en que escribe su primer trabajo importante, el Estudio en Do menor Op. 2 No. 1- y los primeros años del siglo XX -todavía hasta 1903 se pueden encontrar mazurkas y preludios que se enmarcan en este período- ${ }^{36}$ recibe una clara y marcada influencia del romanticismo, de compositores como Chopin, Liszt, Wagner e incluso Schumann.

Las obras de este primer periodo, considerado un romanticismo tardío, se caracterizan por el virtuosismo y el lenguaje poético propios del periodo romántico, aunque revelan una constante experimentación y una búsqueda de un lenguaje armónico propio. ${ }^{37}$ La segunda época, a partir de 1900 -año en que compone una sinfonía inconclusa que contiene la semilla de su Misteriya o Misterium, también inconclusa- y hasta su muerte en 1915, es una época marcada por una búsqueda personal de la trascendencia mística y espiritual, que influye en sus composiciones y lo lleva a construir un lenguaje propio, lleno de símbolos y tendencia a la atonalidad.

La transición a esta segunda época se ve impulsada por su acercamiento a la teosofía, -una doctrina mística religiosa dirigida por Helena Blavatsky que ganó popularidad en la Europa de fines del siglo XIX--, y por su condición sinestésica, que le permitía percibir mediante un sentido estímulos que corresponden a otro sentido. En el caso de Scriabin, su condición sinestésica le permitió asociar colores a cada una de las tonalidades, habilidad que plasmó en su obra Prometeo a través de la utilización de la tastiera di luce, un instrumento diseñado por un fotógrafo de apellido Mozer, que proyectaba un determinado color de acuerdo a la tonalidad que se escuchaba y a la vocal cantada por el coro. ${ }^{38}$ Esta obra constituyó una manifestación filosófica del misticismo musical de Scriabin, que desarrolló de forma obsesiva durante los últimos años de su vida.

Aunque las obras más trascendentes y las que han sido sometidas a análisis minuciosos son precisamente las de este segundo período místico filosófico, la obra que aquí se pretende analizar, el Estudio Op. 8 No. 12 "Patético" compuesto en 1894, corresponde al primer período, que recibe una influencia directa del lenguaje y el sentimiento del romanticismo.

El Estudio Patético se caracteriza por una intensidad sonora producida por variaciones dinámicas y agógicas, además de una dificultad técnica característica

36 Taruskin, R., Oxford history of western music, Vol. 4: Music in the early 20th century, Nueva York, Oxford University Press, 2010, p. 200.

37 Powell, J., "Aleksandr Nikolayevich Skryabin” en The New Grove Dictionary of Music and Musicians, Nueva York, Oxford University Press, 29 tt.

38 Ibidem 
de la obra de Scriabin. La gran técnica que adquirió en la mano izquierda -gracias a que las afectaciones que sufrió su mano derecha le obligaron a practicar sólo con la izquierda-, le permitió componer pasajes de gran dificultad para esa mano, incluso de mayor dificultad que para la mano derecha. Prueba de ello es la complejidad para la mano izquierda en el Estudio Patético, que incluye saltos largos y continuos que requieren gran precisión en la mano. ${ }^{39}$

La mayoría de los análisis a los que ha sido sometido el estudio, como el realizado por Anatole Leikin en su libro The Performing Style of Alexander Scriabin, o la tesis de Laura Lynn Whitehead, llamada Trascendent Sounds: The early piano music of Alexander Scriabin, hacen énfasis en las variaciones de tempo en su ejecución. Scott Holden señala como una edición publicada por el State Memorial Museum of Scriabin analiza estas variaciones de tempo y el uso del rubato a partir de la grabación que el propio Scriabin hizo para la compañía alemana Welte. ${ }^{40}$ El Estudio Patético fue grabado en un piano roll (el número 2073) en febrero de 1910, y en dicha ejecución el tempo oscila entre un valor de 64 y 240 para la nota negra, a pesar de que la partitura indica un valor de entre 100-112 para el mismo valor de nota. Esta amplísima diferencia en el tempo nos lleva a deducir el apasionamiento de la interpretación, que lleva al pianista a exagerar los rubatos y a incluir ritardandos y accelerandos inexistentes en la partitura.

En la primera edición de los 12 Estudios $O p$. 8, a cargo de Mitrofan Petrovich Belaieff, el número 12 tiene ya el adjetivo Patético al inicio de la partitura, añadido por el propio compositor desde el manuscrito (figura 2).$^{41}$ Es posible que dicho adjetivo haya sido añadido sólo como una indicación de interpretación para el pianista, motivada por una vivencia personal o un estado de ánimo melancólico, lleno de impulso o incluso desesperado, y sin un trasfondo de relación entre la estructura del estudio y el concepto del pathos. Esto puede fundamentarse en el hecho de que uno de los factores que influyó en la música de Scriabin fue su nerviosismo, depresión e inestabilidad mental.

La época en la que escribió los Estudios $O p$. 8, estuvo marcada por períodos de desequilibrio mental, como queda de manifiesto en distintas expresiones encontradas en la correspondencia del compositor, citada en la tesis de Whitehead. En una carta de mayo de 1893, dirigida a Natalya Sekerina, uno de sus primeros amores, escribió: "Qué oscuridad en la que vivo. [...] Oh, si tan sólo pudiera ver algo de luz frente a mí" ${ }^{42}$ mientras que en abril de 1895 escribió a Belaieff, el primer editor de los Estudios: "Y oh, ¡mis ánimos extremos! De pronto parece que mi fuerza es ilimitada, todo es conquistado, todo es mío. Después, en un segundo, estoy consciente de mi impotencia. El cansancio y la apatía me dominan. Nunca hay ningún equilibrio en mí" ${ }^{43}$ Estos problemas psicológicos pueden haber contribuido al tono oscuro, agitado y melancólico de composiciones como el Estudio Op. 8 No. 12, o el segundo movimiento de la Sonata para Piano No. 2, Op. 19.44

39 Whitehead, L., Trascendent Sounds: The early piano music of Alexander Scriabin, Canadá, Universidad de Victoria, 2008, p. 48

40 Holden, S., Scriabin: Finding the essence of late Scriabin in his tenth sonata, Lanham, Scarecrow Press, 2012, p. 171.

41 Imagen obtenida del sitio ruso Virtual'nye vystavki (Virtual exhibitions), <http://expositions.nlr.ru/ex_manus/ skriabin/index.php>.

42 Whitehead, L., Trascendent Sounds: The early piano music of Alexander Scriabin, op.cit., p. 20.

43 Ibidem.

$44 \quad$ Ibidem, p. 21. 


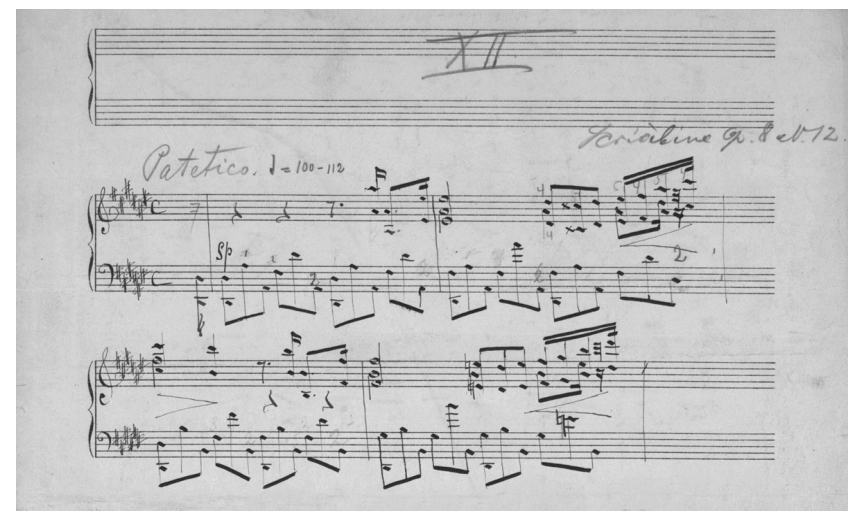

Figura 2. Manuscrito del Estudio Op. 8 No. 12 "Patético", donde se observa que el propio Scriabin añadió el adjetivo "patético" a la obra. Imagen obtenida del sitio ruso Virtual'nye vystavki (Virtual exhibitions)

Sin embargo, ¿es posible hallar figuras retóricas en la partitura del Estudio Patético? Con seguridad podemos hacer interpretaciones respecto a la estructura de la obra y a las frases y motivos que contiene, de la misma manera que Elaine R. Sisman lo hizo con la Sonata Patética de Beethoven. Si observamos la obra en su totalidad podemos detectar con claridad una hipérbole, una amplificación constante del sonido y del ímpetu que llega incluso a la exageración. Los crescendos frecuentes que llevan al clímax apoyado en fortíssimos, constituyen la materia para que la amplificación suceda no sólo en la obra vista como unidad sino también en distintos pasajes. El tema esencialmente está compuesto por gradatio, gradaciones en octavas que aumentan la altura y la intensidad del sonido, y que en secuencia forman la hipérbole que lleva a un clímax temprano (compases 1-7 según la edición de Belaieff). Estas gradaciones van precedidas en la frase por los saltos descendentes de octavas que pueden interpretarse como interrogatio, preguntas que representan momentos de ruptura e inician el discurso.

Según la idea de diferenciación entre ethos y pathos utilizada en la literatura europea del siglo XVIII, las gradaciones en octavas que forman la melodía creciente representan el pathos, es decir, el sentimiento fuerte e impetuoso, mientras que las octavas y saltos de la mano izquierda, que forman la armonía de manera estable y continua, representan el ethos (figura 3). ${ }^{45}$ La relación entre melodía y pathos surge de la intensificación melódica, en el sentido geográfico y dinámico. Geográficamente, o a lo largo del teclado, las octavas suben gradualmente hasta llegar a la octava de fa5, mientras que dinámicamente son cada vez más agudas, desde piano hasta forte. El patrón rítmico que sigue la mano izquierda puede interpretarse como el ethos estable y continuo, ya que no se alteran las figuras rítmicas, manteniéndose en tresillos durante casi toda la pieza.

45 Imagen obtenida del sitio IMSLP: Petrucci Music Library, < http://ks.imslp.net/files/imglnks/usimg/f/ f6/ IMSLP309417-PMLP07375-Skrjabin_op.08_Zwoelf_Etueden.pdf>. Anotaciones sobre la imagen realizadas por Dario D. Aguillón Gutiérrez. 
La estructura AABA de la obra permite una reexposición del tema (compases 9-16), aunque con mayor intensidad al incluir acordes más completos y mayor cantidad de octavas. Al final de la obra vuelve la sección A, incluso con mayor intensidad, y de la misma manera que Sisman lo interpreta en el Grave de la Patética de Beethoven, posiblemente son distintas respuestas a una pregunta original. ${ }^{46} \mathrm{En}$ la sección B (compases 17-32) podemos encontrar nuevamente una serie de gradaciones que esta vez a manera de diálogo (sermocinatio), presentan distintas interrogatio que obtienen respuesta a través de cambios de tonalidad que pueden entenderse como correctio (sustitución de palabras -notas en este caso-, para llamar la atención a través del cambio) (figura 4). ${ }^{47}$

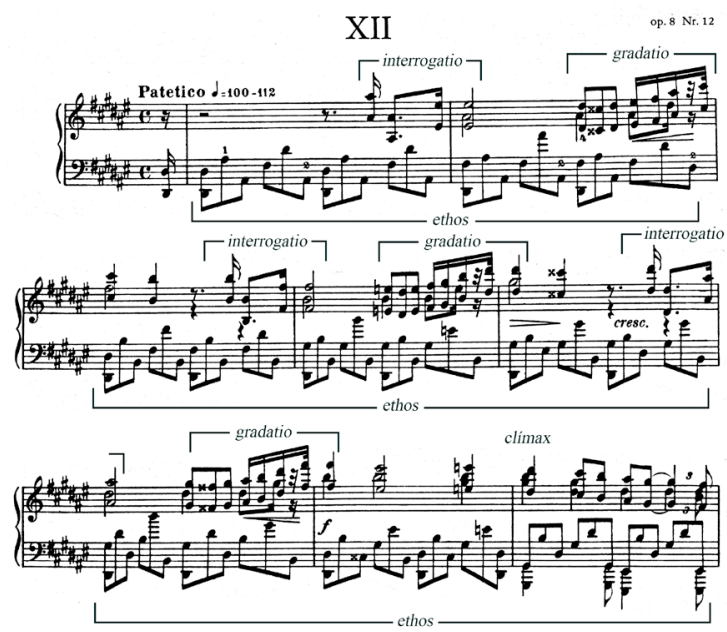

Figura 3. Figuras retóricas en la exposición del tema del Estudio Op. 8 No. 12 "Patético"
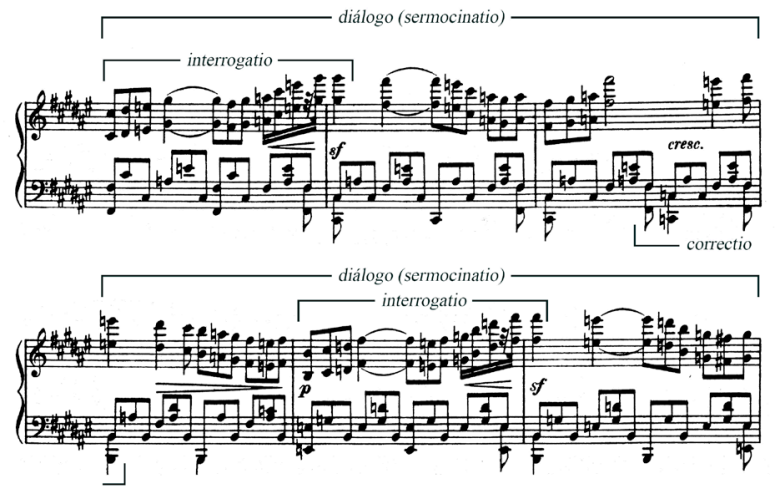

Figura 4. Figuras retóricas en la sección B, donde sucede un diálogo (sermocinatio)

46 Sisman, E., Pathos and the Pathétique: retorical stance in Beethoven's C minor sonata Op. 13, op. cit., p. 101.

47 Imagen obtenida del sitio IMSLP: Petrucci Music Library, < http://ks.imslp.net/files/imglnks/usimg/f/ f6/ IMSLP309417-PMLP07375-Skrjabin_op.08_Zwoelf_Etueden.pdf $>$. Anotaciones sobre la imagen realizadas por Dario D. Aguillón Gutiérrez. 
La sección B termina con un motivo que se repite cuatro veces (compases 31-33) y que anuncia el regreso al tema original a manera de apostrophe, figura que consiste en dirigirse con gran emoción y vehemencia al oyente en un tono patético (figura $5){ }^{48}$ El final de la obra, que regresa al tema en la última sección A (compases 3349), constituye una visión o fantasía (phantasia), es decir, una vívida imagen de una escena ausente que incrementa la intensidad y la pasión, a través del uso de acordes llenos que producen una sonoridad que envuelve.

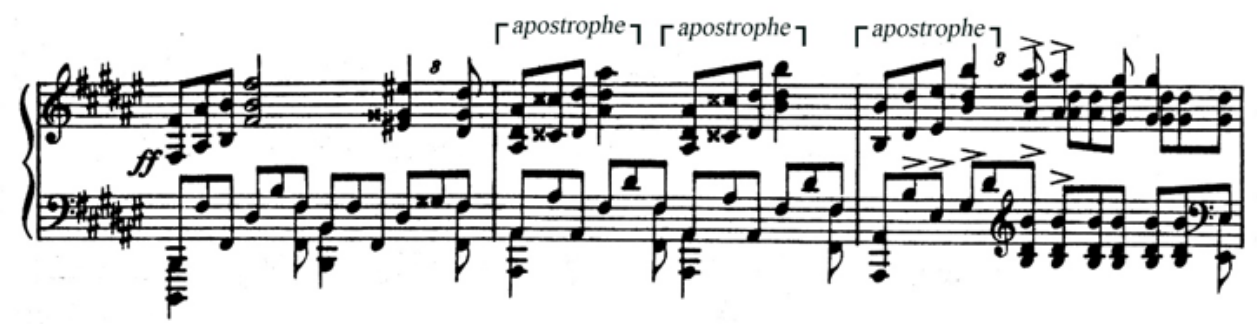

Figura 5. Figuras retóricas en el final de la sección B.

Apostrophes que anuncian el regreso del tema principal

La segunda reexposición del tema en esta última sección, lleva a un clímax cuyos acordes en el bajo forman una gradatio (compases 44-48), que nuevamente gracias a su amplificación y exageración, constituye una hipérbole (figura 6) ${ }^{49} \mathrm{La}$ coda (compases 49-55) comienza con un descenso basado en el motivo inicial de la obra, y después con un ascenso por todo el teclado, desde las notas más graves hasta las más agudas, que rompe el esquema para cerrar con dos acordes en la tonalidad principal.

Es un hecho que el anterior análisis consiste en una interpretación personal respecto a las figuras retóricas asociadas a lo patético que pueden encontrarse en la obra. Esta interpretación está basada en la teoría de la retórica, y en las figuras descritas por distintos autores como Quintiliano, Johann Christoph Adelung y George Campbell -todos citados en la investigación de Sisman- ${ }^{50}$ y busca encontrar fundamentar el carácter patético del estudio de Scriabin, aun cuando no existe evidencia alguna de que el compositor intencionalmente buscara una relación de su obra con el concepto del pathos griego. Sin embargo, el presente análisis aporta a la comprensión y a una interpretación consciente de la obra, para lograr un verdadero discurso basado en el pathos que logre persuadir a su audiencia.

48 Imagen obtenida del sitio IMSLP: Petrucci Music Library, < http://ks.imslp.net/files/imglnks/usimg/f/ f6/ IMSLP309417-PMLP07375-Skrjabin_op.08_Zwoelf_Etueden.pdf>. Anotaciones sobre la imagen realizadas por Dario D. Aguillón Gutiérrez.

49 Imagen obtenida del sitio IMSLP: Petrucci Music Library, < http://ks.imslp.net/files/imglnks/usimg/f/ f6/ IMSLP309417-PMLP07375-Skrjabin_op.08_Zwoelf_Etueden.pdf>. Anotaciones sobre la imagen realizadas por Dario D. Aguillón Gutiérrez.

50 Sisman, E., Pathos and the Pathétique: retorical stance in Beethoven's C minor sonata Op. 13, op. cit., p. 81105 . 


\section{Conclusión}

Es evidente que el concepto del pathos ha sido utilizado en la historia de la música de diversas formas, a veces consciente y profundamente y muchas otras de manera superficial. A pesar de que pudiera pensarse que el profundo interés de Scriabin en la filosofía motivó una real intención de relacionar el Estudio Patético con el concepto filosófico del pathos, esto no es así al ser el estudio una obra temprana, que no está catalogada entre las que recibieron influencia del misticismo musical.
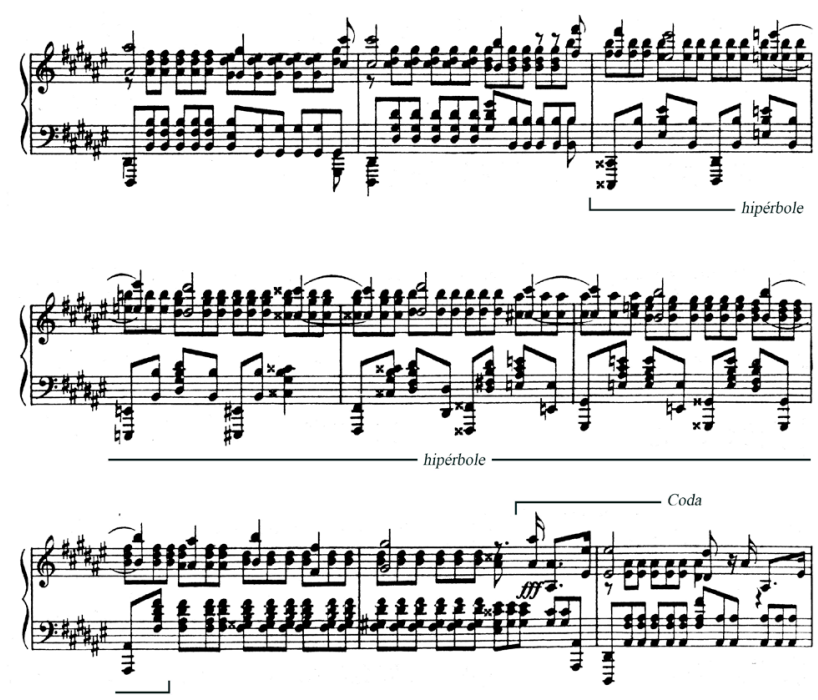

Figura 6. Figuras retóricas en el final de la obra, después de la visión o fantasía o segunda reexposición del tema principal

La obra se puede analizar desde una perspectiva romántica, en un lenguaje todavía tonal que corresponde a una etapa formativa del estilo del compositor, y muy probablemente creada a consecuencia de un episodio dramático causado por sus constantes depresiones y desequilibrios mentales.

Si bien el concepto de lo patético es intrínseco al pathos, no necesariamente todas las obras que portan dicho adjetivo tienen que poseer en su esencia una relación estrecha o una composición consciente basada en el concepto filosófico. Recordemos que la tradición literaria ha transformado el concepto y lo ha llevado a la superficialidad, asociándolo con emociones y sentimientos como la melancolía o la ira, sin necesidad de profundizar en su origen filosófico discursivo.

Sin embargo, el análisis de las obras musicales no debe limitarse a la observación desde la óptica de la teoría de la música, sino que deben buscarse los elementos contextuales, históricos, literarios o filosóficos que permitan enriquecer su interpretación. El conocimiento y comprensión de los elementos formales y estructurales de la obra sin duda ayuda a su correcta ejecución técnica, sin embargo, la comprensión del contexto y las razones por las que fue compuesta, así como las motivaciones del compositor de añadirle ciertos adjetivos o carácter propio, pueden permitir que el intérprete aborde la obra con mayor claridad y con precisión respecto 
a lo que el autor deseaba transmitir. Porque, a fin de cuentas, el discurso musical busca persuadir al oyente, entregarle un mensaje de características únicas que sólo ella, la música, es capaz de entregar, y a través de él producir en el espíritu y en la conciencia una amplísima gama de emociones y sentimientos.

Desde la antigua Grecia el objetivo fundamental de la música ha sido la formación y la transformación, y no hay mejor manera de expresar su capacidad transformadora, que como lo hizo Beethoven hace ya algunos siglos: "La música constituye una revelación más alta que ninguna filosofía".

\section{Referencias bibliográficas}

Anne Coudreuse, «Pathos et pathétique au XVIII ${ }^{e}$ siècle: étude lexicale et statistique», en Studi francesi, 126, 1998, <https://hal.archives-ouvertes.fr/hal-00655161/>.

Aristóteles, Retórica. Libros I y II, Madrid, Editorial Gredos, edición de 1990.

Beristáin, Helena, Diccionario de Retórica y Poética, México, Editorial Porrúa, 1992.

Dissmore, Joshua L., «Baroque Music and the Doctrine of Affections: Putting the Affections into Effect», The Research and Scholarship Symposium, 18 (2017).

Emanuel, Barbara, Rodrigues, Camila y Martins, Marcos, «Rhetoric of Interaction: Analysis of Pathos», en Design, User Experience, and Usability, 1, 2015, Cham: Springer International Publishing <https://www.academia.edu/18235724/Rhetoric_of_ Interaction_Analysis_of_Pathos>.

García Morente, Manuel, Lecciones Preliminares de Filosofía, México, Editorial Porrúa, 1992.

Gutiérrez Heras, Joaquín, Notas sobre notas. México, Consejo Nacional para la Cultura y las Artes, 1998.

Holden, Scott, "Scriabin: Finding the essence of late Scriabin in his tenth sonata», en R.P. Anderson (ed.), The Pianist's Craft: Mastering the Works of great composers, Lanham, Scarecrow Press, 2012.

Leikin, Anatole, The Performing Style of Alexander Scriabin. Burlington, Ashgate, 2011.

Mateos, Agustín, Etimologías grecolatinas del español. Ciudad de México, Editorial Esfinge, 1966.

Orlova, Alexandra, Chaikovsky. Un autorretrato, Madrid, Alianza Editorial, 1994.

Puig, Luisa, «Del pathos clásico al efecto patémico en el análisis del discurso», en Acta Poética, 29 (2), 2008, <https://revistas-filologicas.unam.mx/actapoetica/index.php/ap/ article/view/ 273/274>.

Rowell, Lewis, Introducción a la filosofía de la música, Buenos Aires, Gedisa, 1985.

Schiller, J. Ch. Friedrich, Escritos sobre estética, Madrid, Tecnos, edición de 1991 de Juan M. Navarro Cordón.

Sisman, E., «Pathos and the Pathétique: rethorical stance in Beethoven's C minor sonata Op. 13», en Beethoven Forum, vol. 3, 1994, <http://www.colby.edu/music/saunders/MU397/ Sisman_Pathos.pdf $>$.

Solomon, Maynard, Beethoven, Buenos Aires, Javier Vergara Editor, 1983.

Taruskin, Richard, Oxford history of western music, Nueva York, Oxford University Press, 2010, 5 vols.

The New Grove Dictionary of Music and Musicians, Nueva York, Oxford University Press, 2001, 29 tt. 
Whitehead, Laura L., Trascendent Sounds: The early piano music of Alexander Scriabin, tesis de maestría en Artes, Canadá, Universidad de Victoria, 2008.

Wright, James K, Schoenberg, Wittgenstein and the Vienna Circle, Berna, Peter Lang, 2007.

Referencias electrónicas

Virtual'nye vystavki (Virtual exhibitions), <http:/expositions.nlr.ru/ex_manus/skriabin/ index.php>. 